Y.-H. Chu, N.B. Suntzeff, J.E. Hesser, and D.A. Bohlender, eds.

\title{
The ISM near SN 1987A: Kinematics and 3-D Structure
}

\author{
Arlin P.S. Crotts and Jun Xu \\ Dept. of Astronomy, Columbia University, New York, NY 10027, USA
}

\begin{abstract}
We present two views of the region around SN 1987A: a three-dimensional map of structure revealed by light echoes, and a multicomponent velocity map at $10 \mathrm{~km} \mathrm{~s}^{-1}$ resolution in [N II], correlating the various superbubble structures found in both, with implications for their age and dynamics, as well as the history of the SN progenitor
\end{abstract}

The Large Magellanic Cloud (LMC) exhibits gas shells from $\sim 100 \mathrm{pc}$ to $1000 \mathrm{pc}$ across. A new tool for unfolding the three-dimensional (3D) configuration of these structures seen to overlap in projection is the light echo from SN 1987A. We (Xu, Crotts, \& Kunkel 1995) discuss the large scale interstellar medium (ISM) distribution in the SN foreground, and construct a map of these complicated structures from the light echoes, identifying dust sheets from 310 to $3240 \mathrm{pc}$ in front of the SN. Additionally, we report results from a map of the velocity structure in the same field, revealed by long-slit echelle spectroscopy in [N II] (Xu \& Crotts 1999). This information is tied to the same features seen in the echoes, revealing the kinematics and dynamics of the bubbles.

Significantly, N157C is not just the small shell of $\mathrm{H} \alpha$ filaments north-east of SN1987A. Instead, echoes from $\mathrm{H} \alpha$ structure extending south-west of the SN form a sphere with center of curvature on OB association LH90, at N157C's center, so N157C extends to $\sim 220 \mathrm{pc}$ in diameter. X-ray maps (Wang \& Helfand 1991a) reveal a bubble $\sim 120 \mathrm{pc}$ across, while N157C seen in $21-\mathrm{cm}$ emission extends over $\gtrsim 200$ pc (Kim et al. 1998), nearly coextensive with the echo. The dust coincides with the $\mathrm{H} \mathrm{I}$, but sits outside the X-ray-emitting region.

Two additional bubbles are indicated along the sightline to the SN. Two echoes sit $\sim 1 \mathrm{kpc}$ in front of SN 1987A. These and N157C may form opposite sides of a giant bubble $600 \mathrm{pc}$ across. Dense clouds about $100 \mathrm{pc}$ in front of SN1987A bound a large region evacuated around SN 1987A, and therefore may contain the bubble blown by the $\mathrm{SN}$ and its companions. We also notice components at 269 and $301 \mathrm{~km} \mathrm{~s}^{-1}$ within $\sim 20^{\prime \prime}$ of SN1987A. These structures are probably due to the red supergiant wind, and are treated in a separate paper.

High resolution $\left(10 \mathrm{~km} \mathrm{~s}^{-1}\right)$ [N II] spectra, sampled every 13 arcsec in a $6^{\prime}$ square around SN1987A were obtained on the CTIO $4 \mathrm{~m} /$ Echelle (Xu \& Crotts 1999). Three components, $V_{\text {hel }}=265,277$ and $285 \mathrm{~km} \mathrm{~s}^{-1}$, were identified as $\mathrm{N} 157 \mathrm{C}$, having the same morphology as the echoes corresponding to the shell centered on LH90. Wang and Helfand (1991b) reported X-ray emission in $\mathrm{N} 157 \mathrm{C}$, corresponding to very hot gas; therefore, N157C may be in the energy-conservation stage. The radius of this superbubble is found to expand at $10 \mathrm{~km} \mathrm{~s}^{-1}$, with a lifetime of $6 \times 10^{6}$ years and a total energy of $3 \times 10^{51} \mathrm{ergs}$, determined according to superbubble theory (McCray \& Kafatos 1987): $R_{S}=$ 
$97 p c\left(N_{\star} E_{51} / n_{0}\right)^{1 / 5} t_{7}^{3 / 5}$ (Eqn. 1), and $V_{S}=5.7 \mathrm{~km} \mathrm{~s}^{-1}\left(N_{\star} E_{51} / n_{0}\right)^{1 / 5} t_{7}^{-2 / 5}$ (Eqn. 2). Dividing one equation by the other, $t_{7}=\left(R_{S} V_{S}\right) /\left[\left(5.7 \mathrm{~km} \mathrm{~s}^{-1}\right)(97 \mathrm{pc})\right]$ (Eqn. 3), where $n_{0}$ is the gas atomic density, the age $t_{7}=t / 10^{7} \mathrm{yr}, R_{S}=100 \mathrm{pc}$, and $V_{S}=10 \mathrm{~km} \mathrm{~s}^{-1}$. We estimate $\mathrm{N} 157 \mathrm{C}$ to have $t=6 \mathrm{Myr}$, still very young.

The morphology of $V_{h e l}=235 \mathrm{~km} \mathrm{~s}^{-1}$ component corresponds to the echo $1 \mathrm{kpc}$ in front of the SN, the near side of the $600 \mathrm{pc}$ cavity. With nothing between this structure and $\mathrm{N} 157 \mathrm{C}$, this indicates a bubble expanding at $\sim 40 \mathrm{~km} \mathrm{~s}^{-1}$ relative to the centroid of N157C. We suspect that this $600 \mathrm{pc}$ bubble has blown out of the LMC disk: not only is its wall fragmented, but Wang and Helfand (1991b) report that this region has soft X-ray emission much higher than the background but much lower than other young superbubbles in 30 Doradus. Also, $600 \mathrm{pc}$ is one of the largest superbubbles ever observed in the LMC, and approaches the limit on the superbubble size imposed by LMC disk thickness. McCray and Kafatos (1987) suggest that superbubbles breaking out of the galaxy, distort by gravitational instability, collapse to form giant clouds, and ignite propagating star formation. LH90 may have formed in the collapse of the $600 \mathrm{pc}$ bubble.

If this superbubble blew out in its snow-plow stage, prior to this it was described in terms of the time $\left(t_{c}\right)$ and expansion radius $\left(R_{c}\right)$ beyond which cooling becomes important in the interior e.g. post-adiabatically, according to McCray and Kafatos: $R(t) \approx R_{c}\left(t / t_{c}\right)^{1 / 4}$, for $R_{c}=50 p c \zeta^{-0.9}\left(N_{\star} E_{51}\right)^{0.4} n_{0}^{-0.6}$, and $t_{c}=4 \times 10^{6} \zeta^{-1.5}\left(N_{\star} E_{51}\right)^{0.3} n_{0}^{-0.7}$, where $\zeta \approx 0.3$ is metallicity, $N_{\star}$ is number of stars $\geq 7 M_{\odot}, E_{51}=E / 10^{51}$ ergs, and $n_{0} \approx 0.35$ is gas atomic density. Let's guess $N_{\star}=50$ (like $\mathrm{N} 157 \mathrm{C}$ ) and $E_{51}=1$, hence $t_{c}=160 \mathrm{Myr}$, and $R_{c} \approx 1.3 \mathrm{kpc}$, much bigger than $300 \mathrm{pc}$. Therefore, this bubble was probably hot when it blew out, implying an age at blowout of $\sim 10 \mathrm{Myr}$ from eq. 3 , or $15 \mathrm{Myr}$ using eq. 1 . This appears older than N157C, and may have created LH90.

Two components, $V_{\text {hel }}=255$ and $245 \mathrm{~km} \mathrm{~s}^{-1}$ are identified as the inner major echo $130 \mathrm{pc}$ in front of SN1987A. Two components, 300 and $313 \mathrm{~km} \mathrm{~s}^{-1}$, may be the far side of a bubble in which SN1987A exploded. They do not correspond to any known absorption lines or echoes (which show up mainly in front), hence probably lie behind the SN. If these components form the far side of a bubble surrounding $\mathrm{SN} 1987 \mathrm{~A}$, its radius expands at $30 \mathrm{~km} \mathrm{~s}^{-1}$. If we adopt a $90 \mathrm{pc}$ radius, the bubble's age is $\sim 2 \mathrm{Myr}$ (from eq. 1 ).

We find that the time it took the SN1987A progenitor to move to the current location 300 pc behind $\mathrm{N} 157 \mathrm{C}$ (at $\gtrsim 20 \mathrm{~km} \mathrm{~s}^{-1}$ ) is comparable to the age of $\mathrm{N} 157 \mathrm{C}$ as well as that of the progenitor itself $\left(\sim 10^{7} \mathrm{y}\right)$. This suggests that perhaps LH90 and the progenitor were created in the same previous superbubble collapse. Suggestively, the age the $600 \mathrm{pc}$ giant bubble is consistent with this.

\section{References}

Kim, S., et al. 1998, ApJ, 503, 674

McCray, R., \& Kafatos, M. 1987, ApJ, 317, 190

Wang, Q., \& Helfand, D.J. 1991a, ApJ, 370, 541

Wang, Q., \& Helfand, D.J. 1991b, ApJ, 373, 497

Xu, J., \& Crotts, A.P.S. 1999, ApJ, 511, 262

Xu, J., Crotts, A.P.S., \& Kunkel, W.E. 1995, ApJ, 451, 806 (Erratum: 463, 391) 


\section{Discussion}

David Graff: What is the density of the different clumps?

Crotts: Most of the extinction is in N157C. There is greater extinction in patches of the cloud $1 \mathrm{kpc}$ in front of the $\mathrm{SN}$, but this feature is very non-homogeneous. The wall $100 \mathrm{pc}$ in front of the SN is a few times thinner than N175C. The total amount of material corresponds to about $2 \times 10^{21} \mathrm{~cm}^{-2}$ in $\mathrm{H} \mathrm{I}$. 